\title{
Local Ventromedial Hypothalamus Glucose Perfusion Blocks Counterregulation during Systemic Hypoglycemia in Awake Rats
}

\author{
Monica A. Borg, ${ }^{\star}$ Robert S. Sherwin, ${ }^{\star}$ Walter P. Borg, ${ }^{\star}$ William V. Tamborlane, ${ }^{\ddagger}$ and Gerald I. Shulman* \\ Yale University School of Medicine, ${ }^{*}$ Department of Internal Medicine and ${ }^{\ddagger}$ Department of Pediatrics, New Haven, Connecticut 06520
}

\begin{abstract}
The ventromedial hypothalamic nucleus (VMH) is necessary for the integrated hormonal response to hypoglycemia. To determine the role of the VMH as a glucose sensor, we performed experiments designed to specifically prevent glucopenia in the VMH, while producing hypoglycemia elsewhere. We used awake chronically catheterized rats, in which local VMH glucose perfusion $(100 \mathrm{mM}$ or $15 \mathrm{mM}$ of D-glucose) was combined with a sequential euglycemichypoglycemic clamp. In two control groups the VMH was perfused either with $(a)$ an iso-osmotic solution lacking glucose, or with $(b)$ nonmetabolizable L-glucose (100 mM). During systemic hypoglycemia glucagon and catecholamine concentrations promptly increased in the control animals perfused with either $100 \mathrm{mM}$ L-glucose or the iso-osmotic solution lacking glucose. In contrast, glucagon, epinephrine and norepinephrine release was inhibited in the animals in which the VMH was perfused with D-glucose; hormonal secretion was partially suppressed by the VMH perfusion with $15 \mathrm{mM}$ D-glucose and suppressed by $\sim 85 \%$ when the VMH was perfused with $100 \mathrm{mM}$ D-glucose, as compared with the control groups. We conclude that the VMH must sense hypoglycemia for full activation of catecholamine and glucagon secretion and that it is a key glucose sensor for hypoglycemic counterregulation. (J. Clin. Invest. 1997. 99:361-365.) Key words: VMH • microdialysis • hypoglycemia • glucagon • epinephrine
\end{abstract}

\section{Introduction}

The central nervous system (CNS) ${ }^{1}$ plays an important role in sensing glucopenia and triggering counterregulatory hormone release during hypoglycemia. (1-3). Although a region within or in close proximity to the liver may activate the sympathoadrenal system (4), the CNS appears to be the dominant center responsible for the sensing and integration of hypoglycemic

\footnotetext{
Address correspondence to Gerald I. Shulman, M.D., Ph.D., Yale University School of Medicine, Department of Internal Medicine/Endocrinology, Box 208020, FMP 104, New Haven, CT 06520-8020. Phone: 203-785-5447; FAX: 203-785-6015; E-mail: gerald_shulman@ qm.yale.edu

Received for publication 19 July 1996 and accepted in revised form 5 November 1996.
}

1. Abbreviations used in this paper: CNS, central nervous system; $\mathrm{ECF}$, extracellular fluid; VMH, ventromedial hypothalamic nucleus.

J. Clin. Invest.

(C) The American Society for Clinical Investigation, Inc.

0021-9738/97/01/361/05 \$2.00

Volume 99, Number 2, January 1997, 361-365 signals. This view is best supported by data showing that prevention of intercerebral glucopenia by infusion of glucose into both the carotid and vertebral arteries nearly abolishes counterregulatory hormone responses to systemic hypoglycemia (2, 5). Recent studies suggest that the ventromedial hypothalamus (VMH) may be the specific region in the CNS that is responsible for activation of counterregulatory mechanisms, since focal lesioning in this area abolishes the hormonal response to systemic hypoglycemia (6). Moreover, production of local neuroglycopenia by perfusion of 2-deoxy-glucose into the $\mathrm{VMH}$ is able to trigger the release of counterregulatory hormones, despite systemic normoglycemia (7).

The present study was undertaken to examine whether the converse is also true; namely, can hormonal responses to systemic hypoglycemia be blocked by preventing neuroglycopenia in the VMH, alone? For this purpose, the hypoglycemic insulin clamp technique was combined with bilateral VMH microdialysis in Sprague-Dawley rats; the microdialysis perfusate contained extracellular fluid (ECF) with added glucose. This approach allowed us to selectively prevent hypoglycemia within the chosen brain region, and to test its effect under standardized hypoglycemic conditions.

\section{Methods}

Animals. Male Sprague-Dawley rats were purchased from Charles River Laboratories (Raleigh, NC). Animals were housed in an environmentally controlled room with a 12 -h light/dark cycle, and were maintained on standard ad libitum rat diet (AGWAY Prolab 3000, Waverly, NY) comprising of $22 \%$ protein, $5 \%$ fat, and $51 \%$ carbohydrate (the remaining $22 \%$ consists of ash, crude fiber, and moisture).

VMH microdialysis canulas placement. Rats (mean body weight of $\sim 280 \pm 5 \mathrm{~g}$, range $250-315 \mathrm{~g}$ ) were anesthetized by intraperitoneal injection $(1 \mathrm{ml} / \mathrm{kg}$ ) of a mixture of Xylazine (AnaSed $20 \mathrm{mg} / \mathrm{ml}$; Lloyd Laboratories, Shenadonoah, IA) and Ketamine (Ketaset $100 \mathrm{mg} / \mathrm{ml}$; Aveco Co., Fort Dodge, IA) in a ratio of $1: 2(\mathrm{vol} / \mathrm{vol})$ and placed on a stereotaxic frame. Thereafter, the skull was exposed, and holes were drilled bilaterally in chosen coordinates, through which the guide canulas were lowered slowly into the brain. The stereotaxic coordinates were determined from the atlas of Paxinos and Watson (8). Specifically, VMH canulas were placed by using the coordinates $2.6 \mathrm{~mm}$ posterior and $3.8 \mathrm{~mm}$ lateral in relation to bregma, and the angle of $20^{\circ}$ in relation to horizontal plane passing through bregma and lambda. The canulas were then secured to the skull with stainless steel screws and dental acrylic. Animals were then allowed to recover from the stereotaxic procedure for $12-16 \mathrm{~d}$ before study. One day before each experiment, microdialysis probes of side-by-side design (9) were inserted into the guide canulas. The length of the probes was $10.5 \mathrm{~mm}$, as measured from the bregma-lambda plane. The exposed microdialysis membrane was 1.0-1.5 mm (approximately the size of the VMH), so that we could selectively perfuse this brain region. On the morning of the experiment, the perfusion medium was loaded into 1-ml syringes and delivered at a flow rate of $2.5 \mu \mathrm{l} / \mathrm{min}$ using a Harvard perfusion pump (model 22; Harvard Bioscience). Four groups of rats (two experimental and two controls) were studied. In each the VMH was perfused with a sterile, ascorbate-free, artificial, extracellular fluid so- 
lution (ECF: $135 \mathrm{mmol} /$ liter $\mathrm{NaCl}, 3 \mathrm{mmol} / \mathrm{l} \mathrm{KCl}, 1 \mathrm{mmol} / \mathrm{liter} \mathrm{MgCl}_{2}$, $1.2 \mathrm{mmol} / \mathrm{CaCl}_{2}$, and $2 \mathrm{mmol} /$ liter sodium phosphate buffer to $\mathrm{pH}$ 7.4). The VMH probe was perfused in the first group with $100 \mathrm{mM}$ D-glucose (metabolically active isomer) and in the second group with $15 \mathrm{mM}$ D-glucose. The third and the fourth groups of animals served as controls: The third group had no D-glucose added to the perfusate (i.e., ECF only was perfused into the $\mathrm{VMH}$ ) and the fourth group of rats had $100 \mathrm{mM}$ of the non-metabolizable, L-glucose isomer added to the perfusate. The last group of animals served as a control for the high osmolarity of the perfusate used in groups perfused with D-glucose. High concentrations of glucose were used in the perfusate to ensure that hypoglycemia was prevented throughout the $\mathrm{VMH}$, because the efficiency of glucose delivery through the VMH dialysis system is very low, $(<5 \%)$. To estimate the efficiency of glucose delivery, we performed three in vitro experiments, using the same microdialysis system, where only ECF (without glucose added) was perfused in a $100 \mathrm{mM}$ glucose solution via the VMH microdialysis probe. Under these conditions the effluent from the probe contained only $\sim 4.2$ $\mathrm{mM}$ glucose (or $\sim 4 \%$ of glucose) in the solution. Calculations were made, by extrapolating these data to our animal experiments, considering that in vivo (using identical microdialysis probes, solutions, and flow rates), the efficiency of the microdialysis system would be much lower, and the delivery of glucose to be closer to $1-2 \%$ of the infused glucose, or values that approximate brain ECF glucose levels during euglycemia in the rat which are about $1.5-2.0 \mathrm{mM}$ (10). Given these considerations we estimate that the doses of glucose delivered to the $\mathrm{VMH}$ did not produce hyperglycemia in brain ECF.

At the end of each experiment, the accuracy of probe placement was confirmed histologically by cresyl violet staining. Only those animals that showed bilateral probe placement into the desired brain region were included ( $\sim 25 \%$ of rats failed the histological criteria). Fig. 1 presents a photograph of rat brain with microdialysis guide canulas tracks placed bilaterally in the VMH.

The ability of the microdialysis system to deliver glucose selectively to the $\mathrm{VMH}$, has been validated previously in our laboratory, using radiolabeled 2-deoxy-glucose (7). Based on these data, we assume that the $\mathrm{VMH}$ was specifically affected by dialysis, and that no other adjacent area could be significantly influenced by this manipulation.

Surgical procedures. At 6-8 d before study (i.e., $\sim 7 \mathrm{~d}$ after stereotaxic surgery), animals underwent an additional aseptic surgical procedure for placement of internal jugular vein and carotid artery catheters under intraperitoneal pentobarbital anesthesia (Nembutal,
$35 \mathrm{mg} / \mathrm{kg}$ body wt; Abbott Laboratories, North Chicago, IL). The polyethylene carotid artery catheter was extended to the level of the aortic arch, and the silicone internal jugular vein catheter was advanced to the level of the right atrium. At the end of the procedure, both catheters were flushed and filled with heparin $(42 \mathrm{U} / \mathrm{ml})$ and polyvinylpyrrolidone $(1.7 \mathrm{~g} / \mathrm{ml})$ solution, plugged, tunneled subcutaneously around the side of the neck, and externalized behind the head through a skin incision. Catheters remained sealed until the day of the study. Only those animals that had completely recovered were used in the studies.

Specifically, the recovery of the animals after both stereotaxic and catheter surgery was assessed according to a standard protocol approved by the Yale Animal Care and Use Committee. Briefly, for a period of one week after each surgery, animals, housed in the Yale Animal Facility, were monitored for body temperature, feeding activity and body weight on a daily basis. We defined recovery as: (1) maintaining normal rat body temperature $\left(\sim 37.5^{\circ} \mathrm{C}\right)$; (2) attitude acting normally; and (3) weight gain $\sim 7 \mathrm{~g}$ /day. Any sign of infection (focal, localized to the surgerized areas, or generalized) was noted, and sick animals were excluded from participating in the experiments $(\sim 10 \%)$.

Euglycemic/hypoglycemic clamp. The hyperinsulinemic clamp technique, as adapted for the rat (11), was used to provide a standardized hypoglycemic stimulus. Each of the animals was fasted for $\sim 24 \mathrm{~h}$ before the study and then received a sequential euglycemic-hypoglycemic clamp study. On the morning of the experiment, the catheters were flushed and maintained patent by a slow infusion of saline (20 $\mu \mathrm{l} / \mathrm{min})$ that contained a small amount of heparin $(1-2 \mathrm{U} / \mathrm{ml})$. Animals were fully awake and freely moving about in their cages, untethered. After a 60-min rest period, blood samples were withdrawn for measurement of baseline concentrations of glucose, insulin, glucagon, epinephrine, and norepinephrine. Thereafter, a primed $(2,160 \mathrm{pmol}$ over $1.5 \mathrm{~min})$ continuous infusion $\left(120 \mathrm{pmol} / \mathrm{kg}^{-1} \cdot \mathrm{min}^{-1}\right)$ of porcine insulin (Eli Lilly \& Co., Indianapolis, IN) was initiated and maintained for $150 \mathrm{~min}$. A variable infusion of exogenous glucose was adjusted based on plasma glucose measurements obtained at 5-min intervals to achieve the desired glucose level. During the first $60 \mathrm{~min}$, the rats were maintained at euglycemia (mean plasma glucose 6.4 $\mathrm{mM}$ ). Thereafter, plasma glucose was allowed to fall over $\sim 40 \mathrm{~min}$ to hypoglycemic levels $(\sim 2.5 \mathrm{mM})$ and then clamped at this level for $\sim 45 \pm 5$ min using a variable infusion of exogenous glucose. Experiments were terminated if the plasma glucose $(a)$ fell below $4.5 \mathrm{mM}$ during the last $45 \mathrm{~min}$ of the euglycemic phase and $(b)$ rose above 3.9

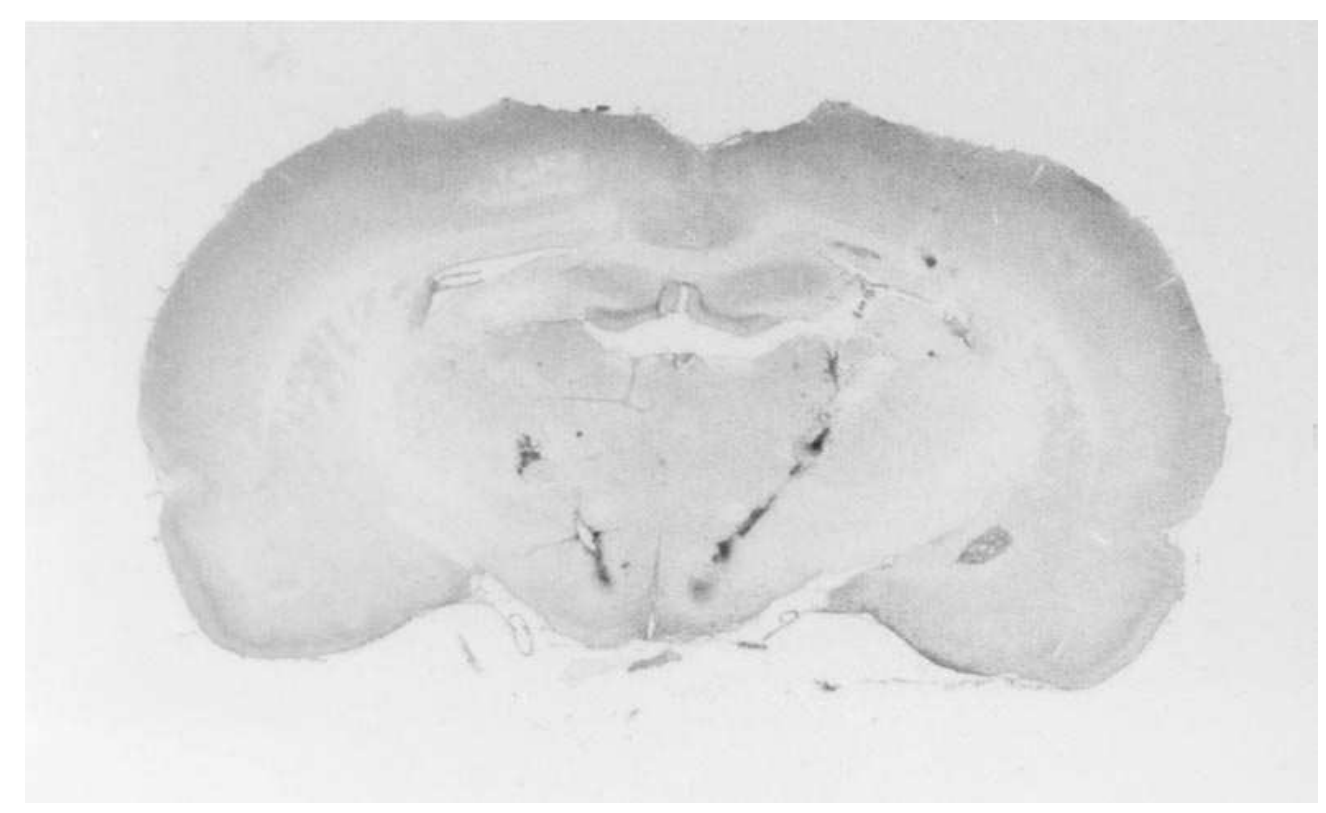

Figure 1. Photograph of the rat brain with the microdialysis guide cannulas tracks placed bilaterally in the VMH. The exposed dialysis membrane was approximately the size of the VMH, that is $1.0-1.5 \mathrm{~mm}$. Three different concentrations of D-glucose $(0,15$, and 100 $\mathrm{mM}$ ) and $100 \mathrm{mM}$ L-glucose were used in the experiment. 
$\mathrm{mM}$ (secondary to glucose infusion), or inadvertently fell below 2.0 $\mathrm{mM}$ during the hypoglycemic phase. Only the studies during which the mean blood glucose level achieved during hypoglycemia was in the range of $2.3-2.7 \mathrm{mM}$ were prospectively analyzed. In the first experimental group of rats, where the VMH was perfused with $100 \mathrm{mM}$ D-glucose, $14 \%$ of animals with proper canula placement (as verified histologically) did not meet these criteria. In the second group (perfused with $15 \mathrm{mM}$ D-glucose) $12 \%$ of animals were excluded. Two control groups (where ECF only or L-glucose perfusion were introduced) had $25 \%$ and $20 \%$ animals excluded, respectively. Histological results and the accuracy of the clamps were the only criteria for excluding or including the clamp studies for the data analysis.

Blood samples for measurement of plasma insulin, glucagon and catecholamines were taken during the euglycemic (30- and 60-min) and hypoglycemic (120-, 135-, and 150-min) phases of the experiments. During blood sampling, dilution by fluid in the dead space of the catheter was avoided by withdrawal of $0.5 \mathrm{ml}$ of blood before sample collection. This volume exceeded the dead space volume by about five fold. A new syringe was then used for the sample collection. Subsequently, the initial syringe was reattached, and the contents reinfused. Blood recently obtained from littermates was transfused during the clamp to quantitatively replace the blood withdrawn during the experiment. The protocol was reviewed and approved by the Yale Animal Care and Use Committee.

Analytical methods and calculations. Plasma glucose was measured in duplicate using a Beckman Glucose Analyzer II (Beckman, Fullerton, CA). Plasma insulin (Binax, South Portland, MA), and glucagon (ICN Biomedicals, Inc., Carson, CA) were determined by a double antibody radioimmunoassay. Insulin measurements during the basal and insulin infusion periods were made using rat and por- cine standards, respectively. Plasma catecholamines were measured with a radioenzymatic method (Amersham Corp., Arlington Heights, IL), as previously described $(6,7)$.

Data are expressed as mean \pm SE. Comparison between the experimental groups was made by ANOVA with a repeated measure design, followed by the Student's $t$ test to localize effects. Glucose infusion rates during the hypoglycemic steps represent average values for the designated time interval. Values for percent suppression of hormonal responses reflect changes from the mean of the peak counterregulatory response (i.e., the mean of the 135 and 150 min data points) results in the control studies.

\section{Results}

At the outset of the study, neither the basal concentrations of glucoregulatory hormones nor the weight of the animals (Table I), were significantly different in the four experimental groups. During the insulin infusion, plasma insulin rose to nearly identical levels, and plasma glucose was comparable during each phase of the study in all groups (Table II and Fig. 2). In the hypoglycemic phase of the study, the desired level of glycemia (i.e., $\sim 2.5 \mathrm{mM}$ ) was achieved after $\sim 45 \mathrm{~min}$.

During euglycemia the concentrations of epinephrine, norepinephrine, and glucagon were not significantly different among the four groups of animals. The effects of the different VMH microdialysis perfusions on counterregulatory hormones are summarized in Fig. 3. In both experimental groups (VMH perfused either with $100 \mathrm{mM}$ or $15 \mathrm{mM}$ D-glucose) hor-
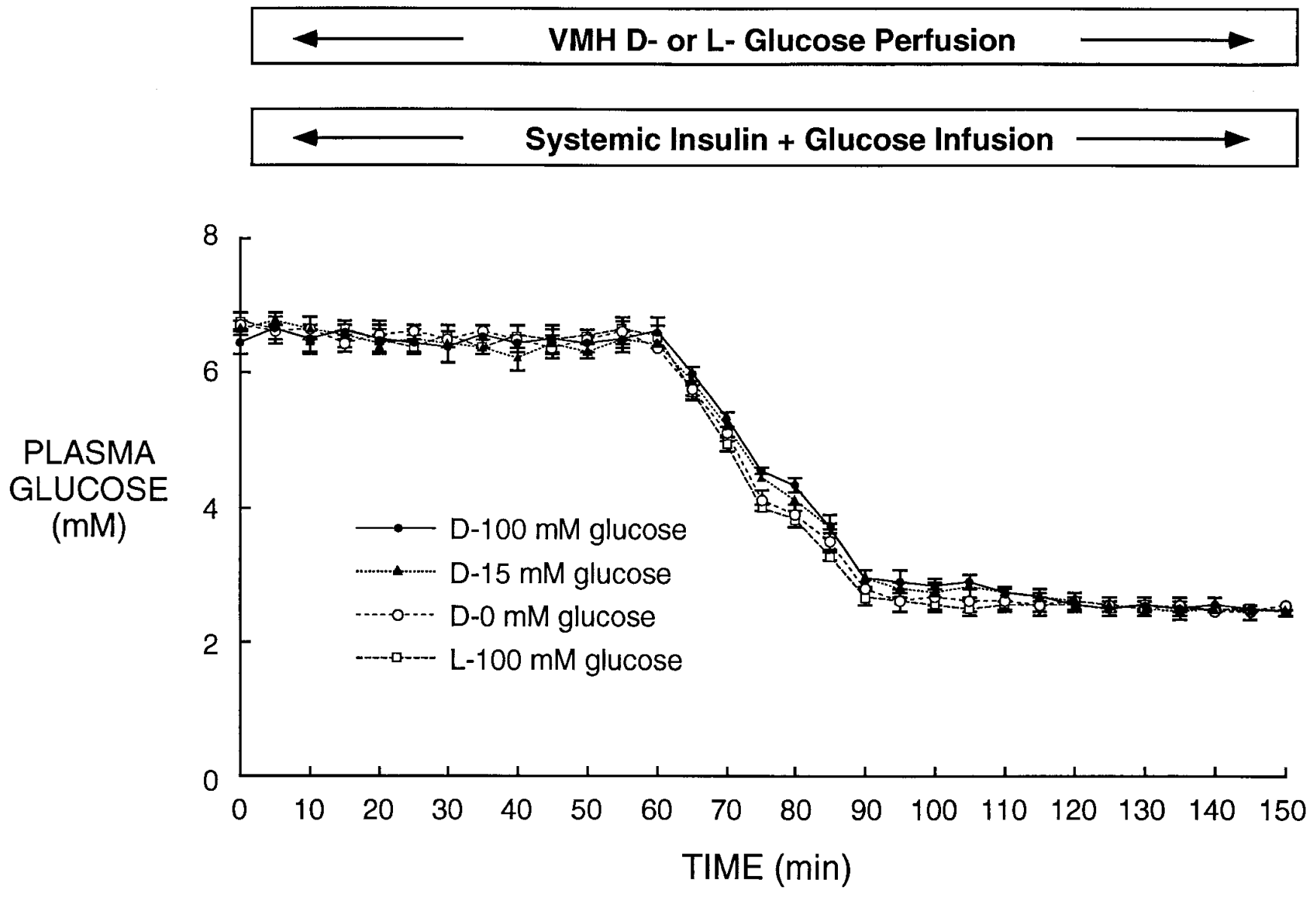

Figure 2. Plasma glucose concentrations during euglycemic-hypoglycemic clamps plus VMH microdialysis perfusions. 
Table I. Basal Hormonal and Weight Data of the Animals Studied

\begin{tabular}{lcccc}
\hline & \multicolumn{4}{c}{ VMH perfusions with } \\
\cline { 2 - 5 } & $\begin{array}{c}100 \mathrm{mM} \\
\text { D-glucose }\end{array}$ & $\begin{array}{c}15 \mathrm{mM} \\
\text { D-glucose }\end{array}$ & $\begin{array}{c}0 \mathrm{mM} \\
\text { D-glucose }\end{array}$ & $\begin{array}{c}100 \mathrm{mM} \\
\text { L-glucose }\end{array}$ \\
\hline & $(n=6)$ & $(n=7)$ & $(n=9)$ & $(n=8)$ \\
Insulin (nM) & $0.11 \pm 0.04$ & $0.12 \pm 0.03$ & $0.13 \pm 0.02$ & $0.12 \pm 0.02$ \\
Glucagon (ng/liter) & $146 \pm 13$ & $152 \pm 17$ & $180 \pm 15$ & $212 \pm 43$ \\
Epinephrine (nM) & $0.7 \pm 0.1$ & $0.6 \pm 0.1$ & $1.2 \pm 0.4$ & $1.1 \pm 0.3$ \\
Norepinephrine (nM) & $1.7 \pm 0.3$ & $1.6 \pm 0.2$ & $2.2 \pm 0.5$ & $2.1 \pm 0.5$ \\
Weight (g) & $295 \pm 20$ & $270 \pm 20$ & $280 \pm 30$ & $285 \pm 25$ \\
& & & & \\
\hline
\end{tabular}

monal responses were diminished, despite a similar degree of systemic hypoglycemia. Specifically, $100 \mathrm{mM}$ D-glucose perfusion into the VMH suppressed the release of epinephrine and norepinephrine by $90 \%$ and glucagon by $75 \%(P<0.05$ as compared with both control groups). The VMH perfusion with $15 \mathrm{mM}$ D-glucose had a less pronounced effect, but also suppressed epinephrine and norepinephrine by $\sim 75 \%(P<0.05$ vs. both control groups) and glucagon by $\sim 45 \%(P<0.05$ only as compared with the VMH perfusion with ECF). In contrast, there was a sharp increase in the concentrations of plasma catecholamines and glucagon in the two control groups, when hypoglycemia was induced.

The diminished counterregulatory hormone responses in the animals receiving VMH D-glucose perfusion was associated with a 3-4-fold higher rate of exogenous glucose infusion than was required in the control studies to maintain the plasma glucose plateau at $2.5 \mathrm{mM}$ (Table II).

\section{Discussion}

This study demonstrates that when hypoglycemia is selectively prevented in the $\mathrm{VMH}$, but not in the remainder of the CNS or elsewhere, the catecholamine response, and to a lesser degree

Table II. Mean Plasma Glucose and Insulin Concentrations during Euglycemia and Hypoglycemia

\begin{tabular}{|c|c|c|c|c|}
\hline & \multicolumn{4}{|c|}{ VMH perfusions with } \\
\hline & $\begin{array}{c}100 \mathrm{mM} \\
\text { D-glucose }\end{array}$ & $\begin{array}{c}15 \mathrm{mM} \\
\text { D-glucose }\end{array}$ & $\begin{array}{c}0 \mathrm{mM} \\
\text { D-glucose }\end{array}$ & $\begin{array}{c}100 \mathrm{mM} \\
\text { L-glucose }\end{array}$ \\
\hline & $(n=6)$ & $(n=7)$ & $(n=9)$ & $(n=8)$ \\
\hline \multicolumn{5}{|c|}{ Euglycemia $(T=0-60 \mathrm{~min})$} \\
\hline Glucose $(\mathrm{mM})$ & $6.4 \pm 0.1$ & $6.4 \pm 0.2$ & $6.5 \pm 0.1$ & $6.4 \pm 0.1$ \\
\hline Insulin (nM) & $4.9 \pm 0.5$ & $4.7 \pm 0.4$ & $4.9 \pm 0.5$ & $5.0 \pm 0.6$ \\
\hline \multicolumn{5}{|c|}{ Hypoglycemia $(\mathrm{T}=105-150 \mathrm{~min})$} \\
\hline Glucose $(\mathrm{mM})$ & $2.5 \pm 0.2$ & $2.5 \pm 0.1$ & $2.4 \pm 0.1$ & $2.4 \pm 0.1$ \\
\hline Insulin (nM) & $5.0 \pm 0.6$ & $4.9 \pm 0.4$ & $5.0 \pm 0.4$ & $5.2 \pm 0.7$ \\
\hline $\begin{array}{l}\text { Exogenous glucose } \\
\text { infusion rate } \\
\left(\mathrm{mg} \cdot \mathrm{Kg}^{-1} \cdot \mathrm{min}^{-1}\right)\end{array}$ & $5.2 \pm 1.2 *$ & $4.4 \pm 1.1 *$ & $1.5 \pm 0.5$ & $1.3 \pm 0.4$ \\
\hline
\end{tabular}

$* P<0.05$. Glucose values were obtained by averaging measurements during the 0-60 and 105-150 min of the study. Insulin data for the euglycemic phase represent the average insulin level of the sample obtained at the end of this phase. Insulin values during hypoglycemia represent the average of measurements obtained at 120 and $150 \mathrm{~min}$.

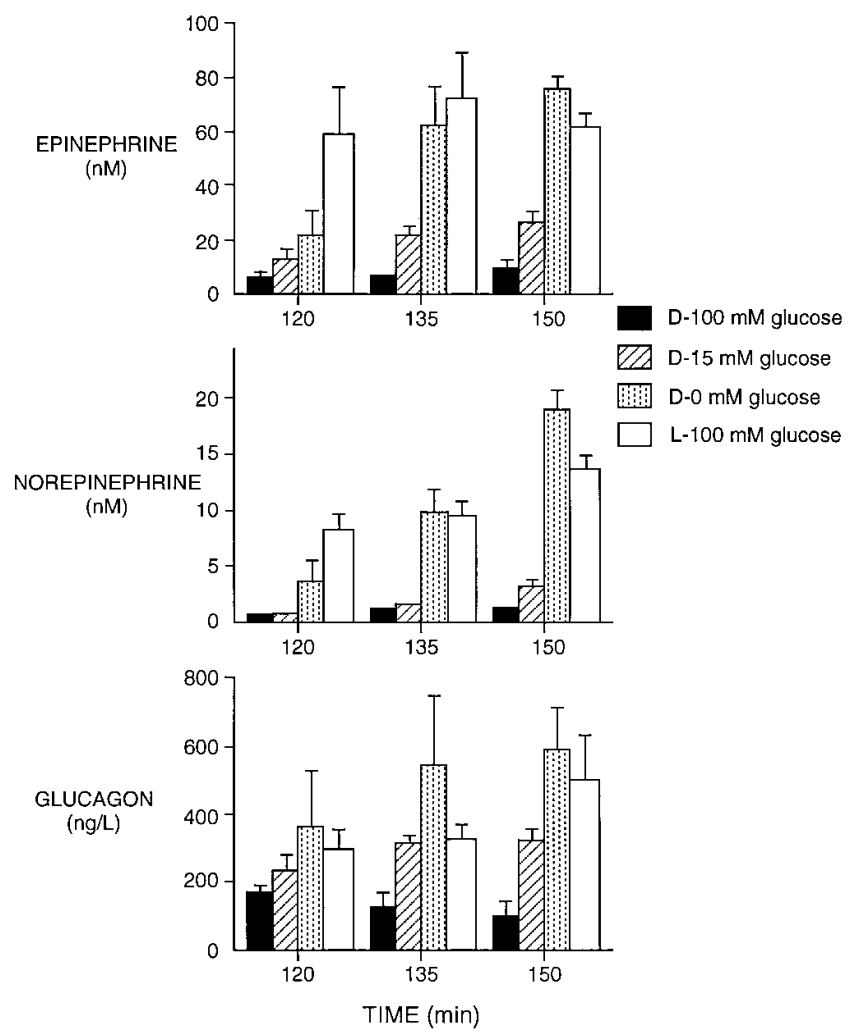

Figure 3. Effect of D-glucose VMH perfusion on the hypoglycemiainduced increment in plasma counterregulatory hormones.

the glucagon response, is reduced in the face of systemic hypoglycemia. No inhibition of counterregulatory hormone release occurred when the VMH was perfused with ECF only (no D-glucose added) or with $100 \mathrm{mM}$ L-glucose or when results from those studies are compared with those of naive rats previously reported (6). In keeping with these findings, the two experimental groups receiving the VMH D-glucose perfusion required more exogenous glucose to maintain the hypoglycemic plateau than did control animals which had intact counterregulatory responses.

While the characteristics of counterregulatory responses during hypoglycemia are well established $(6,7)$, the physiologic events that occur to link glucopenia with counterregulatory hormone secretion remain obscure. Although the bulk of data suggest that the brain plays a critical role in this process, the specific anatomical location of the center for sensing and integration of hypoglycemic signals is uncertain $(5,12)$. In previous studies we performed hypoglycemic clamp studies in bilaterally VMH lesioned rats as well as in rats with lesions in different brain areas (6). A marked inhibition of both the glucagon and catecholamine response to hypoglycemia was evident only in the VMH-lesioned animals, consistent with earlier studies implicating the hypothalamus in the activation of hypoglycemic counterregulation (13-15).

In this study we establish in a more specific and physiological way the critical role that the VMH plays in the counterregulatory response to hypoglycemia. Specifically, these data demonstrate that the catecholamine response to systemic hypoglycemia is blocked, and the glucagon response is attenuated by direct glucose delivery to the VMH. By using the se- 
quential euglycemic-hypoglycemic clamp technique, we avoided the confounding effects of variable glycemic levels among the study groups. Additionally, the bilateral intracranial microdialysis technique allowed us to minimize or to abolish cellular glucopenia in an area of brain restricted to the VMH. Probe placement, while obviously invasive, did not appear to significantly alter the hormonal response to hypoglycemia, since the magnitude of the hormonal response in the control rats reported here was very similar to that reported by our group in naive animals during comparable hypoglycemic clamp studies (6). It is noteworthy that VMH perfusion with $100 \mathrm{mM} \mathrm{D}$-glucose was sufficient to block the sympatho-adrenal and to attenuate pancreatic $\alpha$-cell response to systemic hypoglycemia, whereas only partial inhibition of both glucagon and catecholamine response was seen, when $15 \mathrm{mM}$ glucose was perfused through the probes. While the reason for this discrepancy is uncertain, it is likely that the low efficiency of glucose delivery via the probe may have prevented complete abolition of hypoglycemia throughout all neurons in the $\mathrm{VMH}$ during the $15 \mathrm{mM}$ but not the $100 \mathrm{mM}$ glucose perfusion, especially VMH neurons located at greater distances from the probe. It is unlikely that the inhibition of counterregulatory release induced by perfusion of the VMH with $100 \mathrm{mM}$ D-glucose can be attributed to direct osmotic effects, since perfusion of the VMH with $100 \mathrm{mM}$ L-glucose had no effect on the counterregulatory response. Moreover, in previous experiments, perfusion of $100 \mathrm{mM}$ D-glucose into the VMH had no effect on counterregulatory hormone levels under euglycemic conditions (7).

It should be noted, however, that some data do not support the concept that the dominant glucose sensing neurons are located in the VMH. Studies by Donovan et al. have suggested that peripheral glucosensors may play a role in the activation of hypoglycemia-induced sympatho-adrenal responses (4). Recent studies performed by these investigators have shown that portal euglycemia reduces counterregulatory responses to hypoglycemia, implying that the VMH may not be the only area detecting hypoglycemia $(16,17)$. However, in order to create conditions of euglycemia in the liver, glucose was infused into the portal vein which caused a negative arterial-portal glucose gradient. Since this negative gradient has been shown to be an important feeding signal (18), it is possible that this might have affected these results. On the other hand, the current data suggest that if peripheral signaling is involved, it can be overridden if the hypoglycemic stimulus is eliminated at the level of the VMH. In agreement with this Davis et al. have shown that localized hepatic hypoglycemia is unable to evoke counterregulatory hormone release (19). In addition, Jackson et al. (20) have recently reported that blocking transmission of the vagus nerves innervating the liver had no impact on the counterregulatory response to hypoglycemia. In contrast, when localized glucopenia was induced in the VMH by infusion (via microdialysis) of 2-deoxy-glucose, there was a prompt hormone response even in the face of normoglycemia (7). Taken together, these observations suggest that the $\mathrm{VMH}$ is a key glucose sensor for triggering counterregulation. The cellular mechanisms used by the VMH to sense and transduce the glucose signal remain to be determined.

\section{Acknowledgments}

We are indebted to Dr. David Maggs for his comments and suggestions. We appreciate the assistance of Aida Groszmann and Andrea Belous for their help in the measurements of plasma hormones. We also are grateful to Jenny Sherwin and Kimberly Tishler for their expert technical assistance.

This research was supported by Public Health Service grants R01DK 20495, R01-DK 40936, and P01-DK 45735. Drs. M.A. Borg and W.P. Borg are the recipients of fellowship grants from The Juvenile Diabetes Foundation International.

\section{References}

1. Bernard, C. 1858. Lecons sur la Physiologie et la Pathologie en Systeme Nerveus. J.B. Bailliere, Paris. 549-555.

2. Biggers, D., S. Myers, D. Neal, R. Stinson, N. Cooper, J. Jaspan, P. Williams, A. Cherrington, and R. Frizzel. 1989. Role of brain in counterregulation of insulin-induced hypoglycemia in dogs. Diabetes. 37:7-16.

3. Lautt, W. 1980. Hepatic nerves: a review of their functions and effects. Can. J. Physiol. Pharmacol. 58:105-123.

4. Donovan, C., J. Halter, and R. Bergman. 1991. Importance of hepatic glucoreceptors in sympathoadrenal response to hypoglycemia. Diabetes. 40: $155-158$.

5. Frizzel, R., E. Jones, S. Davis, D. Biggers, S. Myers, C. Connolly, D. Neal, J. Jaspan, and A. Cherrington. 1993. Counterregulation during hypoglycemia is directed by widespread brain regions. Diabetes. 42:1253-1261.

6. Borg, W., M. During, R. Sherwin, M. Borg, M. Brines, and G. Shulman. 1994. Ventromedial hypothalamic lesions in rats suppress counterregulatory responses to hypoglycemia. J. Clin. Invest. 93:1677-1682.

7. Borg, W., R. Sherwin, M. During, M. Borg, and G. Shulman. 1995. Local ventromedial hypothalamus glucopenia triggers counterregulatory hormone release. Diabetes. 44:180-184.

8. Paxinos, G., and C. Watson. 1991. The Rat Brain in Stereotaxic Coordinates. Academic Press, New York. 262.

9. During, M. 1991. In vivo neurochemistry of the conscious human brain: intrahippocampal microdialysis in epilepsy. In Microdialysis in the Neurosciences. T.E. Robinson and J.B. Justice, Jr., editors. Amsterdam, Elsevier. 425-442.

10. During, M., P. Leone, K. Davis, D. Kerr, and R. Sherwin. 1995. Glucose modulates rat substantia nigra GABA releases in vivo via ATP-sensitive potassium channels. J. Clin. Invest. 95:2403-2408.

11. Rossetti, L., D. Smith, G. Shulman, D. Papachristou, and R. DeFronzo. 1987. Correction of hyperglycemia with phlorizin normalizes tissue sensitivity to insulin in diabetic rats. J. Clin. Invest. 79:1510-1515.

12. Frohman, L., and K. Nagai. 1976. Central nervous system-mediated stimulation of glucagon secretion in the dog following 2-deoxy-glucose. Metabolism. 25:1449-1452.

13. Himsworth, R. 1970. Hypothalamic control of adrenaline secretion in response to insufficient glucose. J. Physiol. (Lond.). 206:411-417.

14. Anand, B., G. Chhina, K. Sharma, S. Dua, and B. Singh. 1964. Activity of single neurons in the hypothalamic feeding centers: effect of glucose. Am. J. Physiol. 207:1146-1154.

15. Gisel, E., and D. Innes. 1979. Glycemic responses induced by hypothalamic stimulation. Neuroendocrinology. 28:212-216.

16. Donovan, C., M. Hamilton-Wessler, J. Halter, and R. Bergman. 1994. Primacy of liver glucosensors in the sympathetic response to progressive hypoglycemia. Proc. Natl. Acad. Sci. USA. 91:2863-2867.

17. Hamilton-Wessler M., R. Bergman, J. Halter, R. Wanatabe, and C. Donovan. 1994. The role of liver glucosensors in the integrated sympathetic response induced by deep hypoglycemia in dogs. Diabetes. 43:1052-1060.

18. Pagliassotti, M., L. Holste, M. Moore, D. Neal, and A. Cherrington. 1996. Comparison of the time courses of insulin and the portal signal on hepatic glucose and glycogen metabolism in the conscious dog. J. Clin. Invest. 97:81-91.

19. Davis, S., C. Shavers, D. Neal, E. Allen, and P. Williams. 1995. Hepatic hypoglycemia is unable to initiate counterregulation in conscious dogs. Diabetes (Suppl.). 44:3A:1.

20. Jackson, P., P. Williams, D. Neal, P.-S. Hsieh, J. Hastings, and A. Cherrington. 1996. The effect of vagal cooling on the counterregulatory response to hypoglycemia. Diabetes (Suppl.). 45:2A:221. 